\title{
SOME METABOLIC RESPONSES TO REDUCED BREATHING FREQUENCY DURING CONSTANT LOAD EXERCISE
}

\author{
Jernej Kapus $^{(A, B, C, D, E, F, G)}$, Anton Ušaj ${ }^{(A, B, D, E, G)}$, Venceslav $\operatorname{Kapus}^{(A, B, D, E, G)}$ \\ Laboratory of Biodynamics, Faculty of Sport, University of Ljubljana, Slovenia
}

\begin{abstract}
Introduction: Hypoxia has not been detected only during altitude exercise but also during specific conditions during exercise at sea level, such exercise with reduced breathing frequency (RBF). Training with RBF is often referred to as "hypoxic training". It was thought that, by limiting inspired air, the reduction of oxygen available for muscular work would result and therefore cause muscle hypoxia, similar to that experienced at altitude. Therefore, the aim of this study was to examine the possible effect of RBF on ventilatory parameters, blood gases, oxygen saturation and some metabolic responses during the constant load exercise to exhaustion.

Methods: Eight healthy male subjects performed an incremental cycling test with RBF at 10 breaths per minute. A constant load test with RBF (B10) was then performed to exhaustion at the peak power output obtained during the incremental test. Finally, the subjects repeated the constant load test with the spontaneous breathing (SB) using the same protocol as B10.

Results: RBF during the constant load exercise resulted in a profound reduction in $\mathrm{V}_{\mathrm{E}}$, when compared to the spontaneous breathing, despite significantly increases in $\mathrm{V}_{\mathrm{T}}$. Consequently, there were significantly lower $\mathrm{SO}_{2}$ and $\mathrm{PO}_{2}$ and higher $\mathrm{PCO}_{2}$ during $\mathrm{B} 10$ than during SB. However, there were no significant differences in $\mathrm{VO}_{2}$ and [LA-] between different breathing conditions.

Conclusions: Therefore, it may be concluded that RBF during the constant load exercise induced marked hypoventilation and consequently hypoxia and hypercapnia. However, it seemed that this degree of the breathing reduction did not influence on aerobic metabolism due to unchanged $\mathrm{VO}_{2}$ and [ $\left.\mathrm{LA}^{-}\right]$.
\end{abstract}

Key words: constant load test, reduced breathing frequency, hypoxia, hypercapnia, blood lactate

\section{Introduction}

Hypoxia (reduction in oxygen saturation and lower partial pressure of oxygen in arterial and capillary blood) has not been detected only during altitude exercise but also during specific conditions during exercise at sea level, such exercises with reduced breathing frequency (RBF). Due to strictly techniquedependent breathing, RBF is often used during regular swimming training. During front crawl swimming swimmers could easy manipulate with different breathing patterns. Usually, they take breaths every second stroke cycle. However, they could reduce the breathing frequency with taking breath every fourth, fifth, sixth or eighth stroke cycle. Swimming training with RBF is often referred to as „hypoxic training”. It was thought that, by limiting inspired air, the reduction of the oxygen available for muscular work would result and therefore cause muscle hypoxia, similar to that experienced at altitude [1].

Previous studies demonstrated that RBF during exercise elicits a decrease in pulmonary ventilation $\left(\mathrm{V}_{\mathrm{E}}\right)$ with concomitant increase in tidal volume $\left(\mathrm{V}_{\mathrm{T}}\right)$ [2-7] and systematic hypercapnia. The latter was determined by analysing expired air during the exercise
$[2,3,5-7]$ and by measuring capillary blood sampled during [4] and after the exercise $[8,9]$. However, the influences of this kind of breathing during exercise on other metabolic parameters $\left(\mathrm{O}_{2}\right.$ consumption $-\mathrm{VO}_{2}, \mathrm{CO}_{2}$ production $-\mathrm{VCO}_{2}$, arterial $\mathrm{O}_{2}$ saturation $-\mathrm{SO}_{2}$ ) were insufficient. It seemed that the degree of breathing restrictions, the lung volume during breath holding, the exercise intensity and the type of exercise (swimming, cycling and running) were important factors that determine the subject's response to RBF during exercise. Considering that the constant load exercise to exhaustion and the breathing pattern with significantly influence on subject's performance $[4,7]$ were used in this study for the reduced breathing conditions. Therefore, the purpose of this study was to examine the possible effect of the RBF on ventilatory parameters, blood gases and acid base status and some metabolic responses during the constant load exercise to exhaustion.

\section{Materials and methods \\ Subjects}

Eight healthy male subjects (age $25 \pm 1$ years, height $181 \pm 3 \mathrm{~cm}$, weight $80 \pm 7 \mathrm{~kg}$, peak oxygen uptake 
( $\dot{V}_{2}$ peak) $44.26 \pm 2.93 \mathrm{ml} \cdot \mathrm{kg}^{-1} \cdot \mathrm{min}^{-1}$, forced vital capacity of $5.98 \pm 0.58 \mathrm{l}$ and forced expiratory volume of $4.76 \pm 0.59 \mathrm{lin} 1 \mathrm{~s}$ ) volunteered to participate in this study. None of the subjects were smokers and were free of respiratory disease at the time of the study. The subjects were fully informed of the purpose and possible risks of the study before giving their written consent to participate. The study was approved by the University's Research Ethics Committee.

\section{Procedures}

RBF was defined as 10 breaths per minute and was regulated by a breathing metronome. The breathing metronome was composed of a gas service solenoid valve 24 VDC (Jakša, Ljubljana, Slovenia) and a semaphore with red and green lights. Both were controlled by a micro automation Logo DC 12/24V (Siemens, Munich, Germany). The subjects were instructed to expire and inspire during a $2 \mathrm{~s}$ period of open solenoid valve (the green semaphore light was switched on) and to hold their breath, using almost all lung capacity (breath holding near total lung capacity), for $4 \mathrm{~s}$ when the solenoid valve was closed (the red semaphore light was switched on). Prior to the exercise testing, the subjects were familiarized with breathing through the breathing metronome. After familiarization, each subject performed 4 exercise tests on an electromagnetically braked cycle ergometer Ergometrics 900 (Ergoline, Windhagen, Germany) with pedal cadence at $\sim 60$ revolutions per minute (rpm). Tests were performed in a prescribed order, each of them on a different day.

Preliminary tests. The subjects initially performed an incremental exercise test (IT) to obtain $\dot{\mathrm{V}}_{2}$ peak. The test began at $30 \mathrm{~W}$ and increased by $30 \mathrm{~W}$ every 2 min until volitional exhaustion. $\dot{\mathrm{VO}}_{2}$ peak was defined as the highest $\mathrm{O}_{2}$ uptake averaged over 60-s interval. The subjects then performed an incremental exercise test with RBF (ITB10) to obtain peak power output. Except breathing, the protocol of this test was identical to the protocol of IT. The peak power output was defined as the highest work stage that each subject completed. From these results, the work rate for the constant load test with RBF was chosen for each subject.

Experimental protocol. After preliminary testing, a constant load test with RBF (B10) was performed to exhaustion at the peak power output obtained during ITB10. This test started with a $5 \mathrm{~min}$ warm-up at $50 \mathrm{~W}$. After that, the resistance was increased to match the subject's peak power output and the subject continued to exhaustion. The constant load test was completed by $10 \mathrm{~min}$ of active recovery at $20 \mathrm{~W}$ with the spontaneous breathing. Finally, the subjects repeated the constant load test, however, with the spontaneous breathing (SB). The protocol (intensity and duration) of this test was otherwise identical to the protocol of B10.

\section{Measurements}

During the constant load tests (warm-up, exercise and 10 min recovery; SB and B10), the subjects breathed through a mouthpiece attached to a pneumotachograph. The subject's expired gas was sampled continuously by a V-MAX29 (SensorMedics Corporation, Yorba Linda, USA) metabolic cart for a breath-by-breath determination of respiratory (pulmonary ventilation $\left(\mathrm{V}_{\mathrm{E}}\right)$, end-tidal pressure of oxygen $\left(\mathrm{P}_{\mathrm{ET}} \mathrm{O}_{2}\right)$ and end-tidal pressure of carbon dioxide $\left(\mathrm{P}_{\mathrm{ET}} \mathrm{CO}_{2}\right)$ ) and metabolic (oxygen uptake $\left(\mathrm{VO}_{2}\right)$ and carbon dioxide production $\left(\mathrm{VCO}_{2}\right)$ ) parameters. The pneumotachograph and the $\mathrm{O}_{2}$ and $\mathrm{CO}_{2}$ analysers were calibrated prior to the test with a standard $3 \mathrm{~L}$ syringe and precision reference gases, respectively. For further statistical analysis, breath-by-breath data were averaged for each 10-s interval. During the constant load tests (warm-up, exercise and 10 min recovery), oxygen saturation $\left(\mathrm{SO}_{2}\right)$ was measured using a TruStat ${ }^{\mathrm{TM}}$ Pulse Oximeter (Datex-Ohmeda, Madison, USA). The pulse oximeter is an indirect oximetry measuring instrument, which displays $\mathrm{SO}_{2}$ every $4 \mathrm{~s}$. The ear probe was attached to the earlobe after cleaning the area with alcohol.

Measures of parameters of capillary blood gases and acid base status included partial pressure of blood $\mathrm{O}_{2}\left(\mathrm{PO}_{2}\right)$ and $\mathrm{CO}_{2}\left(\mathrm{PCO}_{2}\right)$ and $\mathrm{pH}$ during the warmup and in the second and fourth minute during the exercise. Capillary blood samples $(60-80 \mu \mathrm{l})$ were taken by micro puncture from an earlobe. Earlobe capillary blood was arterialized by the application of hyperemic cream (Finalgon, Boehringer-Ingelheim, Reims, France) at least 20 min before the first capillary sample. Earlobe samples were collected in heparinized glass capillary tubes and introduced into a blood gas analyser ABL5 (Radiometer, Copenhagen, Denmark) for gas analysis at $37^{\circ} \mathrm{C}$. Blood lactate concentration ([LA $]$ ) was measured during the warm-up and in the first, third and fifth minute during the exercise. In addition [LA] was measured also in the second and fourth minute during the recovery after the exercise. Capillary blood samples $(60-80 \mu \mathrm{l})$ were taken by micro puncture from a hyperemied earlobe. Blood samples for measuring [ $\mathrm{LA}^{-}$] were diluted in a LKM41 lactate solution (Dr. Lange, Berlin, Germany) and analysed using the MINI8 (Dr. Lange, Berlin, Germany) photometer.

\section{Statistics}

Breath-by-breath data were average for each 10-s interval. For comparing the respiratory and metabolic parameters and $\mathrm{SO}_{2}$ between two different breathing conditions the data at the defined time during the exercise (the statistically analysed results are marked with standard deviations in the following figures) were used. The results were presented as average values and 
standard deviations (SD). The paired t test was used to compare the data measured during the constant load tests between two different breathing conditions. Statistical significance was accepted at the $P \leq 0.05$ level. All statistical parameters were calculated using the statistics package SPSS (version 15.0, SPSS Inc., Chicago, USA) and the graphical statistics package Sigma Plot (version 9.0, Jandel, Tübingen, Germany).

\section{Results}

Table 1 depicts $\mathrm{VO}_{2}$ responses to incremental cycle ergometry in relation to $\mathrm{VO}_{2}$ responses in subjects during the incremental test with RBF.

Data depicted in table 2, describes the average intensity and time to exhaustion in B10 and yielded $191 \mathrm{~W}( \pm 32 \mathrm{~W})$ and $566 \mathrm{~s}( \pm 332 \mathrm{~s})$, respectively. By design, the intensity and duration of B10 and SB were identical. The data of breathing and metabolic parameters and $\mathrm{SO}_{2}$ measured in the first 320 seconds were statistically analysed due to minimum time to exhaustion obtained in B10 (Table 2).

Figure 1 demonstrates that, RBF during the constant load exercise resulted in a profound reduction in $\mathrm{V}_{\mathrm{E}}(P \leq 0.01)$, when compared to the spontaneous breathing, despite significantly increases in $\mathrm{V}_{\mathrm{T}}$ $(P \leq 0.01)$. This marked hypoventilation induced significantly lower $\mathrm{VCO}_{2}$ during 80 to 120 second of the exercise in B10 in comparison with the corresponding period in the $\mathrm{SB}$ trial $(P \leq 0.01$; Fig. 2$)$. Figure 3 demonstrated that, $\mathrm{P}_{\mathrm{ET}} \mathrm{O}_{2}$ and $\mathrm{P}_{\mathrm{ET}} \mathrm{CO}_{2}$ were significantly lower and higher, respectively, during $\mathrm{B} 10$ than during $\mathrm{SB}(P \leq 0.01)$. In addition, there were significant differences between $\mathrm{SB}$ and $\mathrm{B} 10$ in $\mathrm{SO}_{2}$, measured from 80 seconds to the end of the exercise $(P \leq 0.01$ and 0.05 ; Fig. 4$)$.

The results of parameters of blood gases and acid base status are given in the table 3 .

Table 1. Individual $\mathrm{VO}_{2}$ responses to incremental bicycle exercise in two different breathing conditions

\begin{tabular}{|c|c|c|c|c|c|c|c|c|c|c|c|c|c|}
\hline \multirow{2}{*}{$\begin{array}{l}\text { sub- } \\
\text { ject }\end{array}$} & & \multicolumn{12}{|c|}{ power output (W) } \\
\hline & & 0 & 30 & 60 & 90 & 120 & 150 & 180 & 210 & 240 & 270 & 300 & 330 \\
\hline \multirow{2}{*}{1} & IT & 2,86 & 7,72 & 10,14 & 13,68 & 16,44 & 21,77 & 24,69 & 28,37 & 31,68 & 35,75 & & \\
\hline & ITB10 & 5,00 & 9,26 & 12,11 & 13,80 & 17,13 & 20,75 & 23,65 & 27,12 & 29,81 & & & \\
\hline \multirow{2}{*}{2} & IT & 3,63 & 8,44 & 12,03 & 15,30 & 19,97 & 23,36 & 27,37 & 31,67 & 36,19 & 41,90 & 47,35 & \\
\hline & ITB10 & 5,55 & 11,93 & 13,83 & 16,53 & 21,22 & 24,03 & 28,57 & & & & & \\
\hline \multirow{2}{*}{3} & IT & 3,40 & 8,16 & 11,58 & 15,11 & 19,48 & 23,01 & 27,66 & 32,37 & 35,97 & 40,19 & 45,08 & \\
\hline & ITB10 & 4,70 & 8,27 & 13,71 & 16,48 & 21,03 & 24,56 & 28,26 & 31,64 & & & & \\
\hline \multirow{2}{*}{4} & IT & 3,79 & 8,26 & 11,83 & 15,06 & 17,84 & 20,30 & 23,99 & 28,33 & 32,55 & 36,25 & 39,97 & \\
\hline & ITB10 & 5,51 & 9,67 & 11,73 & 15,15 & 17,20 & 19,19 & & & & & & \\
\hline \multirow{2}{*}{5} & IT & 3,80 & 8,13 & 12,36 & 16,95 & 20,10 & 26,54 & 28,02 & 31,91 & 35,71 & 39,84 & 44,52 & \\
\hline & ITB10 & 4,97 & 9,31 & 13,07 & 15,31 & 18,92 & 21,24 & & & & & & \\
\hline \multirow{2}{*}{6} & IT & 3,80 & 8,68 & 11,50 & 15,68 & 17,68 & 22,08 & 24,78 & 27,98 & 32,68 & 34,69 & 40,10 & 44,63 \\
\hline & ITB10 & 5,22 & 10,28 & 15,25 & 16,54 & 20,06 & 23,54 & 26,49 & 28,94 & & & & \\
\hline \multirow{2}{*}{7} & IT & 4,04 & 8,26 & 11,84 & 15,77 & 18,06 & 22,62 & 25,60 & 29,01 & 33,01 & 36,68 & 42,56 & 46,73 \\
\hline & ITB10 & 4,01 & 8,84 & 12,50 & 14,77 & 18,18 & 18,13 & 22,98 & & & & & \\
\hline \multirow{2}{*}{8} & IT & 3,84 & 9,24 & 12,06 & 15,60 & 19,75 & 23,60 & 27,78 & 31,75 & 36,75 & 43,12 & & \\
\hline & ITB10 & 4,61 & 9,74 & 12,81 & 15,40 & 19,06 & 23,67 & 26,85 & 29,45 & & & & \\
\hline
\end{tabular}

Table 2. Individual values of power output and time to exhaustion during B10

\begin{tabular}{|c|c|c|}
\hline subject & power output $(\mathrm{W})$ & duration (s) \\
\hline 1 & 240 & 420 \\
\hline 2 & 180 & 317 \\
\hline 3 & 210 & 358 \\
\hline 4 & 150 & 1158 \\
\hline 5 & 150 & 354 \\
\hline 6 & 210 & 408 \\
\hline 7 & 180 & 479 \\
\hline 8 & 210 & 4032 \\
\hline
\end{tabular}



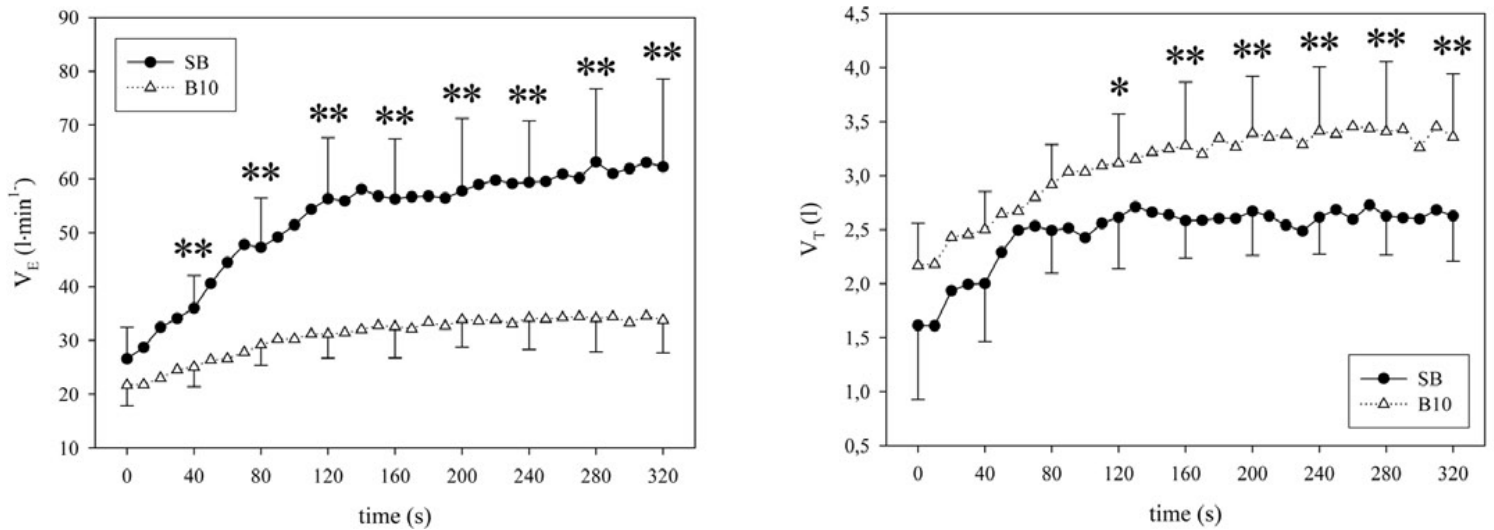

Figure 1. $V_{E}$ and $V_{T}$ during $S B$ (closed circles) and $B 10$ (open triangles) $\left(^{*}-P \leq 0.05 ;{ }^{*}-P \leq 0.01\right)$
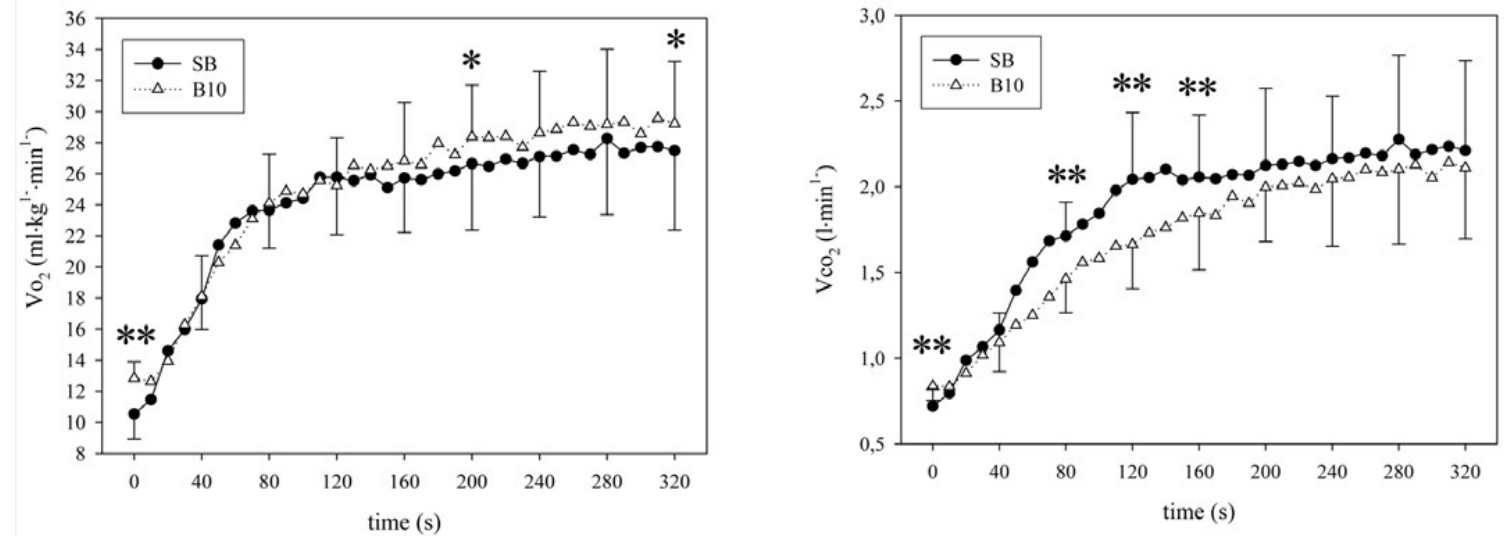

Figure 2. $\mathrm{VO}_{2}$ and $\mathrm{VCO}_{2}$ during $\mathrm{SB}$ (closed circles) and $\mathrm{B} 10$ (open triangles) $\left({ }^{*}-P \leq 0.05 ;{ }^{*}-P \leq 0.01\right.$ )
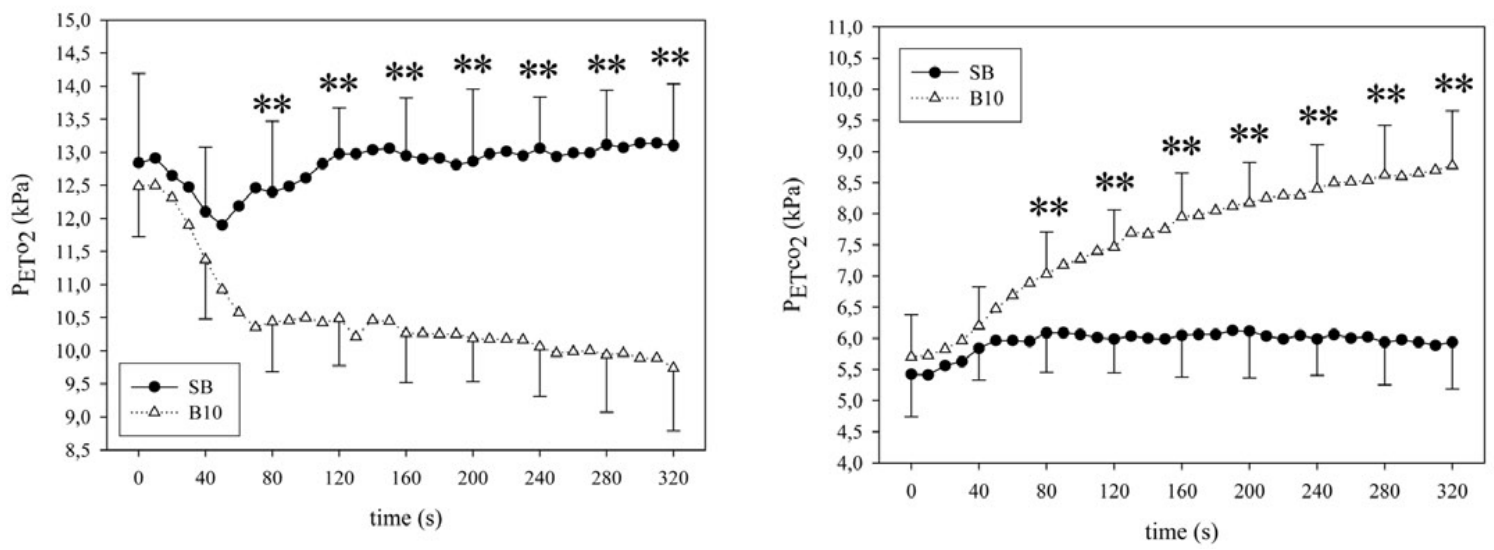

Figure 3. $P_{E T} \mathrm{O}_{2}$ and $P_{E T} \mathrm{CO}_{2}$ during $S B$ (closed circles) and $\mathrm{B} 10$ (open triangles) $\left(^{* *}-P \leq 0.01\right)$

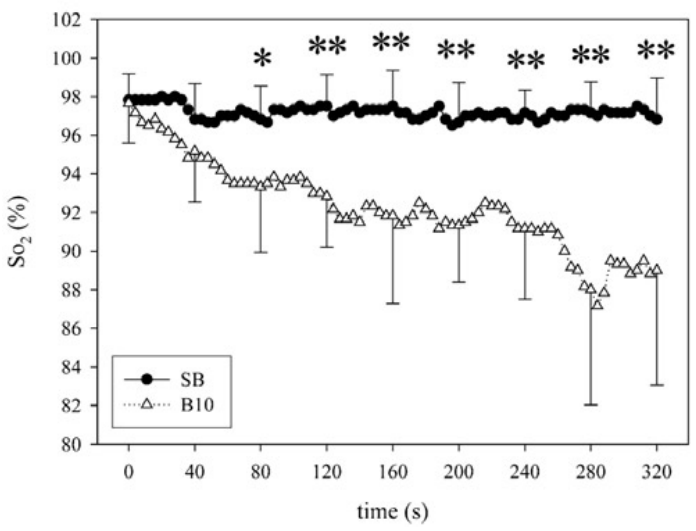

Figure $4 . \mathrm{SO}_{2}$ during the constant load exercise with $\mathrm{SB}$ (closed circles) and with $\mathrm{B} 10$ (open triangles) $\left(^{*}-P \leq 0.05 ;{ }^{* *}-P \leq 0.01\right.$ ) 
Table 3. Comparisons of the parameters of blood gases and acid base status during the warm-up and exercise between two different breathing conditions

\begin{tabular}{|c|c|c|c|}
\hline & & SB & $\mathrm{B} 10$ \\
\hline \multirow{3}{*}{$\mathrm{pH}$} & warm-up & $7.40(0.02)$ & $7.43(0.04)$ \\
\hline & 2. minute during exercise & $7.38(0.02)$ & $7.34(0.01)^{\star *}$ \\
\hline & 4. minute during exercise & $7.36(0.04)$ & $7.29(0.02)^{* *}$ \\
\hline \multirow{3}{*}{$\mathrm{PO}_{2}(\mathrm{kPa})$} & warm-up & $11.1(1.0)$ & $11.3(0.9)$ \\
\hline & 2. minute during exercise & $11.3(0.8)$ & $9.6(0.7)^{* *}$ \\
\hline & 4. minute during exercise & $11.5(0.9)$ & $9.2(1.0)^{* *}$ \\
\hline \multirow{3}{*}{$\mathrm{PCO}_{2}(\mathrm{kPa})$} & warm-up & $5.2(0.5)$ & $5.3(0.6)$ \\
\hline & 2. minute during exercise & $5.8(0.6)$ & $6.4(0.4)^{*}$ \\
\hline & 4. minute during exercise & $5.7(0.7)$ & $6.8(0.5)^{* *}$ \\
\hline
\end{tabular}

${ }^{*}$ and ${ }^{* *}$ denote $P \leq 0.05$ and 0.01 , respectively between the exercises in two different breathing conditions.

Table 4. Comparison of the [LA-] values during the warm-up, exercise and recovery after the exercise between two different breathing conditions

\begin{tabular}{|l|l|c|c|}
\hline & & SB & B10 \\
\hline \multirow{4}{*}[\mathrm{LA}^{-}]{$(\mathrm{mmol} / \mathrm{l})$} & warm-up & $1.3(0.3)$ & $1.3(0.3)$ \\
\cline { 2 - 4 } & 1. minute during the exercise & $1.3(0.3)$ & $3.4(0.4)$ \\
\cline { 2 - 4 } & 3. minute during the exercise & $3.1(1.0)$ & $3.5(0.9)$ \\
\cline { 2 - 4 } & 5. minute during the exercise & $4.5(1.8)$ & $5.1(1.9)$ \\
\cline { 2 - 4 } & 2. minute after the exercise & $4.6(2.5)$ & $4.7(2.8)$ \\
\cline { 2 - 4 } & 4. minute after the exercise & $4.1(2.5)$ & $4.1)$ \\
\hline
\end{tabular}

There were significant differences in $\mathrm{pH}, \mathrm{PO}_{2}$ and $\mathrm{PCO}_{2}$ measured during the exercise between two different breathing conditions ( $P \leq 0.05$ and 0.01 ).

Table 4 demonstrates that there were no significant differences in $\left[\mathrm{LA}^{-}\right]$between SB and B10 measured during warm-up, exercise and recovery.

\section{Discussion}

This study was designed to examine the possible effect of RBF on ventilatory parameters, blood gases and acid base status and some metabolic responses during the constant load exercise to exhaustion. Despite higher $\mathrm{V}_{\mathrm{T}}$ during $\mathrm{B} 10, \mathrm{RBF}$ induced significant lower $\mathrm{V}_{\mathrm{E}}$ in comparison with the spontaneous breathing conditions (Fig. 1). A reduction of approximately $48 \%$ in $\mathrm{V}_{\mathrm{E}}$ was in accordance with the results of previous studies in which 10 breaths per minute was used for the reduced breathing conditions [7]. Furthermore, similar reduction in $\mathrm{V}_{\mathrm{E}}$ was reported when swimmers reduced their breathing frequency during swimming from usual taking a breath every second stroke cycle to taking a breath every fifth [2] or sixth [5] stroke cycle.

Lower $\mathrm{P}_{\mathrm{ET}} \mathrm{O}_{2}, \mathrm{SO}_{2}$ and $\mathrm{PO}_{2}$ during $\mathrm{B} 10$ compared to $\mathrm{SB}$ indicated the hypoxia condition as result of RBF during the constant load exercise (Fig. 3 and 4, Table 3). These results were similar to the results of previous studies in which dry land activities, such us cycle ergometry [4, 7], treadmill running [10] and an exercise on arm crank ergometer [11], were used as an experimental exercise. On the contrary, studies concerning RBF during swimming failed to demonstrate a reduction in oxygen saturation either by analysing expired air during exercise $[2,3,5]$ or by sampling capillary blood after exercise $[8,9]$. Considering that hypoxia has been detected during cycling but not after swimming, the timing of measurement may be the reason for the apparent difference in response to RFB. Indeed, Kapus et al. recently confirmed this hypothesis [12]. They obtained that, due to marked post-exercise hyperventilation, measurements of blood gas parameters analyzed $15 \mathrm{~s}$ after the cessation of exercise did not accurately reflect the condition during exercise with RBF [12].

The theory behind "the hypoxic training" was that RBF produces greater anaerobic response to exercise due to the limited oxygen delivery [13]. This could be indicated by a lower $\mathrm{VO}_{2}$ and by a higher [LA]. However, present study showed no significant differences in these two parameters between SB and B10, despite hypoxia conditions during B10 (Fig. 4, Table 2). These results indicated that an aerobic metabolism was not impeded by RBF during the constant load exercise. According to these results it could be argued that the 
training with RBF elicit aerobic adaptations similar to the high altitude training [4]. On the contrary, in some previous studies RBF during exercises induced lower $\mathrm{VO}_{2}$ in comparison with the spontaneous breathing $[3,6,7,11]$. It seemed that higher degree of a breathing reduction would be sufficient to decrease $\mathrm{VO}_{2}$. However, it is doubtful if the subjects could maintain more reduced breathing during exercise due to distress that they had already reported at the end of B10. To our knowledge, the hypothesis of higher [LA'] during the exercise with RBF has never been confirmed yet. However, some previous studies obtained higher $\left[\mathrm{LA}^{-}\right]$during recovery after the exercise with RBF than after the exercise with the spontaneous breathing $[7,9$, 10]. Considering that, it has been suggested that, the lactate efflux from working muscles was inhibited due to hypercapnia occurred during the exercise with RBF [7]. However in accordance with West et al., results of present study demonstrated that RBF did not alter $\left[\mathrm{LA}^{-}\right]$during and after the exercise [6].

In accordance with previous studies, the marked hypoventilation during $\mathrm{B} 10$ induced higher $\mathrm{P}_{\mathrm{ET}} \mathrm{CO}_{2}$ and $\mathrm{PCO}_{2}$ in comparison with SB (Fig. 3, Table 3). When subjects were allowed to breath spontaneous, their $\mathrm{V}_{\mathrm{E}}$ increased and consequently $\mathrm{P}_{\mathrm{ET}} \mathrm{CO}_{2}$ remained near warm-up levels during the constant load exercise. However, during $\mathrm{B} 10$ increase in $\mathrm{V}_{\mathrm{E}}$ was prevented and consequently $\mathrm{CO}_{2}$ elimination was lower than during $\mathrm{SB}$. Considering lower $\mathrm{VCO}_{2}$, Lee et al. concluded that $\mathrm{CO}_{2}$ was retained in muscle, plasma and erythrocytes during the constant load exercise with RBF [14]. These conditions were reflected in lower $\mathrm{pH}$ during B10 in comparison with SB. Considering obtained results the training with RBF should be termed "hypercapnic training" $[2-4,15]$. It could be suggested that this kind of training during regular swimming practise could improve tolerance to high alveolar $\mathrm{CO}_{2}[2,15]$ and consequently adapt swimmer to swim with fewer breaths. Latter suggestion was recently confirmed [16]. Swimmers decreased their breathing frequency during a maximal 200 meters front crawl with an optional breathing pattern, due to the training with RBF (taking a breath every fourth stroke cycle) during front crawl swimming.

\section{Conclussion}

It may be concluded that the reduced breathing frequency ( 10 breaths per minute) during the constant load exercise induced marked hypoventilation and consequently hypoxia and hypercapnia. However, it seemed that this degree of the breathing reduction did not influence on aerobic metabolism due to unchanged $\mathrm{VO}_{2}$ and $\left[\mathrm{LA}^{-}\right]$.

\section{Acknowledgments}

Research was supported by grant from RR-10-13.

\section{References}

1. Kedrowski V. Hypoxic training. Swim Tech 1979; 13: 55-66.

2. Dicker SG, Lofthus GK, Thornton NW, et al. Respiratory and heart rate responses to controlled frequency breathing swimming. Med Sci Sports Exerc 1980; 12: 20-3.

3. Holmer I, Gullstrand L. Physiological responses to swimming with a controlled frequency of breathing. Scand J Sports Sci 1980; 2: 1-6.

4. Sharp RL, Williams DJ, Bevan L. Effects of controlled frequency breathing during exercise on blood gases and acidbase balance. Int J Sports Med 1991; 12: 62-5.

5. Town GP, Vanness JM. Metabolic responses to controlled frequency breathing in competitive swimmers. Med Sci Sports Exerc 1990; 22: 112-6.

6. West SA, Drummond MJ, VanNess JM, et al. Blood lactate and metabolic responses to controlled frequency breathing during graded swimming. J Strength Cond Res 2005; 19: 772-6.

7. Yamamoto Y, Mutoh Y, Kobayashi H, et al. Effects of reduced frequency breathing on arterial hypoxemia during exercise. Eur J Appl Physiol 1987; 56: 522-7.

8. Kapus J, Ušaj A, Kapus V., et al. The influence of reduced breathing during front crawl swimming on some respiratory and metabolic values in blood. KinSi 2002; 8: 14-8.

9. Kapus J, Ušaj A, Kapus V, et al. The influence of reduced breathing during swimming on some respiratory and metabolic values in blood. KinSi 2003; 9: 12-7.

10. Matheson GO, McKenzie DC. Breath holding during intense exercise: arterial blood gases, $\mathrm{pH}$, and lactate. J Sports Med Phys Fitness 1989: 1947-52.

11. Stager JM, Cordain L, Malley J, et al. Arterial desaturation during arm exercise with controlled frequency breathing. Med Sci Sports Exerc 1985; 17: 227.

12. Kapus J, Ušaj A, Kapus V, et al. The difference in respiratory and blood gas values during recovery after exercise with spontaneous versus reduced breathing frequency. J Sports Sci Med 2009; 8: 452-7.

13. Counsilman JE. Hypoxic and other methods of training evaluated. Swim Tech 1974; 8: 19-26.

14. Lee C, Cordain L, Sockler J, et al. Metabolic consequences of reduced frequency breathing during submaximal exercise at moderate altitude. Eur J Appl Physiol 1990; 61: 289-93.

15. Peyrebrune M, Robinson J, Lakomy H, et al. Effects of controlled frequency breathing on maximal tethered swimming performance. In: Chatard JC., ed. Proceedings of the $9^{\text {th }}$ World Symposium Biomechanics and Medicine in Swimming. Saint-Etienne: Laboratoire de Physiologie and Service d' Exploration Fonctionnelle Cardio-respiratorie et de Medecine du Sport, 2003: 289-94.

16. Kapus J, Ušaj A, Kapus V, et al. The influence of training with reduced breathing frequency in front crawl swimming during a maximal 200 meters front crawl performance. KinSi 2005; 11: 17-24.

Received: October 30, 2009

Accepted: March 25, 2010

Published: March 31, 2010

Address for correspondence:

Jernej Kapus

Faculty of Sport, Gortanova 22, 1000 Ljubljana, Slovenia

E-mail: nejc.kapus@fsp.uni-lj.si

Tel: +38615207796

Fax: +38615207730

Anton Ušaj: anton.usaj@fsp.uni-lj.si

Venceslav Kapus: vene.kapus@fsp.uni-lj.si

\begin{tabular}{lll}
\hline Authors' contribution & B - Data Collection & D - Data Interpretation \\
A - Study Design & C - Statistical Analysis & E - Manuscript Preparation
\end{tabular}

